It is true that the patients we recruited from inpatients admitted with bronchiectasis were more likely to have severe clinical characteristics and poorer FEV1 [8]. However, since the comparisons between both groups in this study were at the same stage, the results still have significant clinical value. In addition, the data from pulmonary function tests were collected when the patients were relatively stable.

Finally, all of the patients with bronchiectasis were routinely asked to sign a consent form when they were admitted to the hospital in our department. Patients signed the consent form to authorise follow-up every 3 months through face-to-face interviews, or telephone interviews if that was not convenient. The reason and date of the exacerbation were recorded according to their medical records. Our study sought to observe the impact of asthma on bronchiectasis exacerbation in a real-world setting. The diagnosis and treatment of bronchiectasis concomitant with asthma remains challenging, and more accurate spirometric and scoring diagnostic methods are needed.

0 @ERSpublications

Asthma increases the risk of bronchiectasis exacerbation; however, more evidence is needed http://ow.ly/dWm0300OcFj

Bei Mao ${ }^{1,2,3}$, Jia-Wei Yang ${ }^{1,2,3}$, Hai-Wen $\mathrm{Lu}^{1}$ and Jin-Fu $\mathrm{Xu}^{1,2}$

${ }^{1}$ Dept of Respiratory and Critical Care Medicine, Shanghai Pulmonary Hospital, Tongii University School of Medicine, Shanghai, China. ${ }^{2}$ Dept of Medicine, Soochow University, Suzhou, China. ${ }^{3}$ These authors contributed equally.

Correspondence: Jin-Fu Xu, Dept of Respiratory Medicine, Shanghai Pulmonary Hospital, No. 507 Zhengmin Road, Shanghai, 200433, China. E-mail: jfxucn@gmail.com.

Received: May 022016 | Accepted: May 262016

Conflict of interest: None declared.

\title{
References
}

1 Mao B, Yang JW, Lu HW, et al. Asthma and bronchiectasis exacerbation. Eur Respir J 2016; 47: 1680-1686.

2 Guan WJ, Gao YH, Xu G, et al. Bronchodilator response in adults with bronchiectasis: correlation with clinical parameters and prognostic implications. J Thorac Dis 2016; 8: 14-23.

3 Pasteur MC, Bilton D, Hill AT. British Thoracic Society guideline for non-CF bronchiectasis. Thorax 2010; 65: 577.

4 Naidich DP, McCauley DI, Khouri NF, et al. Computed tomography of bronchiectasis. J Comput Assist Tomogr 1982; 6: 437-444.

5 Borak J, Lefkowitz RY. Bronchial hyperresponsiveness. Occup Med 2016; 66: 95-105.

6 Global Initiative for Asthma. Global Strategy for Asthma Management and Prevention. www.goldcopd.it/materiale/ 2015/GOLD_Report_2015.pdf Date last accessed: April 2016. Date last updated: 2015.

7 Mao B, Lu HW, Li MH, et al. The existence of bronchiectasis predicts worse prognosis in patients with COPD. Sci Rep 2015; 5: 10961.

8 Guan WJ, Gao YH, Xu G, et al. Characterization of lung function impairment in adults with bronchiectasis. PloS One 2014; 9: e113373.

\section{Obstructive sleep apnoea and bone health}

\author{
To the Editor:
}

We thank LiguORI et al. [1] for sharing their interesting study about patients suffering from obstructive sleep apnoea (OSA) and their tendency towards lower bone mineral density (BMD) in the lumbar spine and femur compared with control subjects matched for age, body mass index and physical activity. The researchers defined "osteopenia" as a T-score value $<-1$ SD and "osteoporosis" as a T-score value $<-2.5 \mathrm{SD}$. They also suggested that OSA could be detrimental to $\mathrm{BMD}$, resulting in osteopenia and osteoporosis. However, there are some concerns with this study.

First, the mean \pm SD age of the subjects included in this study was $51.17 \pm 11.82$ years in the OSA population and $51.10 \pm 11.68$ years in the control group, which means that many of the subjects were $<50$ years old. The author classified these subjects according to T-score, which is the BMD value compared with a healthy subject of the same sex in who is at peak BMD. However, according to the recent consensus of the International Society for Clinical Densitometry [2], when researching males $<50$ years old, a Z-score should be used, representing a value that can be compared with those of subjects matched for age and sex. A Z-score of -2.0 or lower is defined as "below the expected range for age". We suggest that the authors 
should divide the subjects into two groups, older than and younger than 50 years of age, and use T- or Z-scores in the different age groups, respectively. In addition, the authors should not use "osteopenia" or "osteoporosis" to define subjects $<50$ years old.

Moreover, fracture is the most serious consequence of osteoporosis. BMD might be used to predict fractures but the sensitivity is very low. To better understand the effect of OSA on bone health, fracture rate might be a more useful marker. A previous study showed that hypoxia during sleep might be a risk predictive factor for falls and fractures in elderly men [3]. The result of LiGUORI et al. [1] suggests that OSA might be a risk factor for predicting fractures, in addition to low BMD. However, this hypothesis should be confirmed using a clinical study.

$@$ ERSpublications

To better understand the effect of OSA on bone health, fracture rate might be a more useful marker than BMD http://ow.ly/h2vv3018gIg

Jia-Yu Zhong and Ling-Qing Yuan

Institute of Metabolism and Endocrinology, The Second Xiangya Hospital, Central South University, Changsha, China.

Correspondence: Ling-Qing Yuan, Institute of Metabolism and Endocrinology, The Second Xiangya Hospital, Central South University, 139 Middle Renmin Road, Changsha, Hunan 410011, China. E-mail: allenylq@hotmail.com

Received: May 022016 | Accepted: May 262016

Support statement: This work was supported by funding from the National Basic Research Program Programme of China (2014CB942903) and the National Natural Science Foundation of China (81270962). Funding information for this article has been deposited with the Open Funder Registry.

Conflict of interest: Disclosures can be found alongside this article at erj.ersjournals.com

\section{References}

1 Liguori C, Mercuri NB, Izzi F, et al. Obstructive sleep apnoea as a risk factor for osteopenia and osteoporosis in the male population. Eur Respir J 2016; 47: 987-990.

2 International Society for Clinical Densitometry. 2015 ISCD Official positions - Adult. www.iscd.org/official-positions/ 2015-iscd-official-positions-adult/ Date last accessed: October 21, 2015.

3 Cauley JA, Blackwell TL, Redline S, et al. Hypoxia during sleep and the risk of falls and fractures in older men: the Osteoporotic Fractures in Men sleep study. J Am Geriatr Soc 2014; 62: 1853-1859.

Eur Respir J 2016; 48: 1248-1249 | DOI: 10.1183/13993003.00870-2016 | Copyright (CERS 2016

From the authors:

We thank J-Y. Zhong and L-Q. Yuan for their comments on our paper investigating bone mineral density (BMD) in male obstructive sleep apnoea (OSA) patients [1]. In our report, published in the European Respiratory Journal [1], we documented that male patients with severe OSA (apnoea-hypopnoea index $>30$ events $\cdot \mathrm{h}^{-1}$ ) show a severely decreased BMD in the lumbar spine and femur compared with male controls matched for age, body mass index and physical activity. We also documented that OSA patients present an increased risk for osteopenia and osteoporosis. In keeping with our findings, Chi-squared analysis corrected for continuity showed that T-scores consistent with osteopenia/osteoporosis were more frequent in OSA patients compared with controls at all regions of the lumbar spine and at the femur neck, upper femur neck and Ward triangle [1].

In agreement with the recent consensus from the International Society for Clinical Densitometry (ISCD) [2], in their correspondence Zhong and Yuan suggested that Z-score should be used when research about BMD is performed in males younger than 50 years of age. Although we agree with their suggestions, we doubt that there is actually an agreement about the definition of osteopenia/osteoporosis in heterogeneous populations of patients ranging from young-adults to the elderly. Accordingly, since we included subjects ranging from age 30 to 78 years in our study in order to consecutively evaluate patients affected by severe OSA referred to our Sleep Medicine Centre, we used T-scores for defining osteopenia and osteoporosis taking our cue from previous studies evaluating dual-energy X-ray absorptiometry measurement of BMD in the lumbar spine and femur in groups of men patients ranging from young to older ages [3,4]. Although the official positions of the ISCD highlighted that the use of Z-scores, not T-scores, are preferred in males younger than 50 years of age, especially in children, these criteria are clinically useful at an individual level, but do not define the direction for researchers investigating large groups of patients of all ages. However, in keeping with the proposition by Zhong and Yuan, we are performing a further investigation of BMD in young-adult male 